Moura-Vieira, M.E., Luderitz-Hoefel, M.G. \& Réal-Collado, J.T. (2021). El “desierto digital”: repercusiones de la COVID-19 en la Educación en España y Brasil. Revista Electrónica Interuniversitaria de Formación del Profesorado, 24(2), 181-191.

\title{
El “desierto digital”: repercusiones de la COVID-19 en la Educación en España y Brasil
}

\author{
Maria Edna Moura Vieira ${ }^{1}$, Maria da Graça Luderitz Hoefel ${ }^{2}$, José Tomas Réal Collado ${ }^{3}$ \\ 1Secretaria de Estado de Educación del Distrito Federal/Brasil y Instituto de Investigación \\ Sanitaria/INCLIVA-Valencia/ES, 2 Universidad de Brasília/Brasil, 3 Instituto de Investigación \\ Sanitaria/INCLIVA-Valencia/ES
}

\section{Resumen}

Con la aparición de la COVID-19, la forma de hacer y pensar la educación mediatizada por las tecnologías digitales, surgió con una fuerza repentina y nunca experimentada de la forma en que la vimos ocurrir en el año 2020. Así, la nueva realidad educativa se evidencia en la distancia de los cuerpos y la distancia en los procesos de enseñanza y aprendizaje, que ha tenido numerosos impactos en la práctica diaria de la educación. Esta investigación busca conocer las percepciones de los profesores/as de educación básica en España y Brasil, sobre las repercusiones de la COVID-19 en la educación. Se trata de una investigación descriptivaexploratoria, con enfoque cualitativo, que realizó 12 entrevistas semiestructuradas a profesores/as de educación básica de ambos países. Los resultados indican que la incorporación abrupta de tecnologías digitales provocó un gran sufrimiento a los docentes, debido a la falta de formación para ellos, falta de condiciones mínimas de trabajo, aparición de nuevas demandas profesionales y psicológicas, así como diferencias generacionales en las relaciones con las tecnologías.

Palabras clave

Educación; educación básica; tecnologías digitales; COVID 19 . 


\title{
The "digital wasteland": the Covid-19 repercussions in Education in Spain and in Brazil
}

\begin{abstract}
With the emergence of Covid-19, the way of doing and thinking education permeated by digital technologies came up with sudden and never before seen strenght as we experienced in the year of 2020. With it, the new educational reality is highlighted in the distance between the bodies and the distance in teaching and learning processes, which has had numerous impacts in the daily practice of education. This investigation seeks to know the Basic Education professors and lecturers perceptions in Spain and Brasil, about the repercussions of Covid-19 in Education. It is a descriptive-exploratory investigation, with qualitative focus, that performed 12 semi-structured interviews to Basic Education teaching staff from both countries. The results indicate that the abrupt incorporation of digital technologies lead to a great suffering to teachers, on the account of a lack of training, lack of minimum working conditions, emergence of new professional and psychological demands, as well as generational differences in the relationships with the technologies.
\end{abstract}

\section{Key words}

Education; basic education; digital technologies; Covid-19.

\section{Introducción}

Con la aparición del SARS-CoV-2, Coronavirus (COVID-19) a principios de 2020, el mundo se enfrenta ahora al mayor desafío del siglo XXI - evitar el colapso total de sus sistemas de salud y estructuras socioeconómicas (ILO, 2020, p. 7).

La pandemia mundial derivada de la COVID-19, declarada por la (Organización Mundial de la Salud (OMS) el 11 de marzo de 2020), tuvo un impacto especial en el sistema educativo, que comenzó con la suspensión de la actividad educativa presencial en todos los ciclos, grados, cursos y niveles de educación en el contexto mundial (UNESCO, 2020)

En el contexto educativo, esto se desarrolló de tal manera que es muy difícil mantener su status quo, con necesidades inmediatas de nuevos arreglos estructurales y pedagógicos, mientras que su importancia se reafirma en un marco estructural de la sociedad.

Para reducir la transmisión del Coronavirus, muchos gobiernos han adoptado el distanciamiento social. Las instituciones educativas tuvieron que suspender las clases presenciales y la mayoría de ellas dieron continuidad a los procesos educativos mediante la enseñanza a distancia o no presencial.

La forma de hacer y pensar la educación, mediatizada por el entorno digital, surgió con una fuerza repentina y nunca experimentada de la forma en que la vimos ocurrir en el año 2020. La tecnología digital se ha convertido en el dispositivo estructurador de la educación, que proporciona formación en línea, a distancia o remota y diferentes formas de comunicarse, interactuar y relacionarse. 
Con ello, la nueva realidad educativa se evidencia en el distanciamiento de los cuerpos y la distancia en los procesos de enseñanza y aprendizaje, entre las generaciones de las tecnologías digitales (estudiantes) y las tecnologías analógicas (profesores/as).

La rapidez con que todo se dio y ha estado ocurriendo, en este período de COVID-19, ha hecho el tiempo sin tiempo, un tiempo suspendido, un tiempo impredecible. La velocidad con la que se presentan las necesidades puede ser un obstáculo para una conexión significativa entre los eventos y las necesidades reales de la sociedad.

En ese sentido, intentaremos identificar lo que la pandemia está aportando al aprendizaje y las necesidades de la práctica educativa a partir de las experiencias de los profesoreslas de España y Brasil, con el fin de comprender las principales repercusiones y sustentar la discusión en torno a la superación futura de los obstáculos de la formación.

Con este fin, proponemos contribuir a la reflexión sobre las siguientes preguntas: ¿Cómo los gobiernos de España y Brasil abordaron la educación en el contexto de la COVID-19? ¿Cómo están tratando los profesores/as de los dos países el actual contexto educativo donde ocurre un distanciamiento de los cuerpos y unos desgastes debido al confinamiento y las medidas restrictivas impuestas en el aula? ¿Ha sido posible construir un vínculo entre los profesores/as y estudiantes? ¿Los profesores/as se sienten preparados para enfrentarse a ese nuevo contexto educativo mediatizado por el entorno digital?

\section{Educación y COVID-19 en el contexto de España y Brasil: impactos y reflexiones}

La educación es un derecho fundamental que ayuda no sólo al desarrollo personal, sino también al desarrollo de los países. Las repercusiones de la educación son extensas y profundas, impactando en todas las áreas de nuestras vidas. A través de ella garantizamos nuestro desarrollo social, económico y cultural.

En este sentido, la educación es un elemento de suma relevancia en cualquier momento, y especialmente en tiempos de crisis sanitaria, como la que vive el mundo en este año de 2020, cuando, según la UNESCO (2020) alrededor de 190 países de todo el mundo tuvieron que cerrar sus escuelas y universidades para evitar la propagación del coronavirus. Esta situación obligó a 1.500 millones de estudiantes a quedarse en casa.

Conscientes de su importancia y la gravedad del contexto de la pandemia, muchos países han discutido y adoptado mecanismos para asegurar la convivencia y la conexión escolar, a pesar de la distancia causada por el confinamiento social. Las escuelas fueron las primeras instituciones que no pudieron funcionar, ya que son uno de los espacios sociales con mayor intercambio y movilidad de sujetos de diferentes edades, donde puede producirse una contaminación masiva.

En España, desde que la Organización Mundial de la Salud (OMS, 2020) declaró que la situación relativa al coronavirus COVID-19 era una emergencia de salud pública de importancia internacional, se adoptaron una serie de medidas con el fin de proteger la salud y la seguridad de los ciudadanos, contener el avance de la enfermedad y fortalecer el sistema de salud pública. Estas medidas se ampliaron a la luz de la situación generada por la evolución de la pandemia en todo el territorio nacional por parte del Gobierno mediante el Real Decreto 463/2020 de 14 de marzo (ESPAÑA, 2020a) y por los artículos 1 y 2 del Real Decreto 555/2020, de 5 de junio (ESPAÑA, 2020b).

En consecuencia, el año escolar de la educación básica 2019/2020 en España, que comenzó en septiembre de 2019, se celebró de forma presencial (de septiembre de 2019 a principios 
de marzo de 2020) y a distancia (desde marzo hasta junio de 2020). Y el nuevo año escolar 2020/2021, que comenzó en septiembre de 2020, se inició de forma presencial, pero con medidas restrictivas que hacen que la práctica educativa no sea la misma, tales como: distancia mínima, uso de mascarillas, medidas de higiene que incluyen limpieza/higienización del aula dos veces durante el turno escolar, lavado de manos, entre otras.

También están las restricciones que interfieren directamente en la práctica pedagógica/didáctica del profesor/a y su estado emocional, como la orientación de no trabajar en grupo, no poder tener contacto físico, como el abrazo, entre otras.

En Brasil, a diferencia de España, el Gobierno Federal no adoptó una directriz consistente para bajar la curva epidémica, proteger la salud y la seguridad de los ciudadanos. Sobre todo contener el avance de la enfermedad o fortalecer el sistema de salud pública, incluso en relación con las actividades escolares. Según Said (2020) el presidente Jair Bolsonaro reafirmó su política neoliberal en el contexto de la pandemia y relativizó la amenaza del virus, criticó las medidas de confinamiento social y siempre ha defendido el mantenimiento de todas las actividades económicas y sociales, incluidas las escuelas, sin adoptar ningún plan nacional de lucha contra la COVID-19.

A pesar de eso, la mayoría de los gobiernos estatales y municipales han optado por el cierre total de las escuelas para reducir las posibilidades de que los estudiantes se conviertan en vectores del virus para sus familias. Sin el apoyo del gobierno federal, que desde el principio adoptó una política negacionista, lo que dificultó que los gobiernos estatales tomaran medidas efectivas para proteger la salud y la seguridad de los ciudadanos.

Según datos de la Federación Nacional de Escuelas Privadas (FENEP, 2020), las actividades pedagógicas presenciales de las escuelas públicas de Brasil se reanudaron primero en el Estado de Amazonas (agosto de 2020) y seguidamente el Estado de Río Grande do Sul (septiembre de 2020). En el Estado de Pará, el gobierno autorizó clases en algunas regiones. En los demás Estados, las actividades escolares todavía no son presenciales y no tienen una fecha establecida de regreso.

En este contexto, el escenario que se presenta, tanto en España como en Brasil, como en todo el mundo, es complejo y lleno de nuevas necesidades para el universo educativo. De hecho, la pandemia parece haber puenteado, paradójicamente, la importancia de la educación como motor del desarrollo humano, así como las necesidades de formación del profesorado para enfrentar demandas intrínsecas e incertidumbres históricas.

De acuerdo con estas reflexiones, Morin (2020, p.2), alertan sobre la importancia de la educación en el mundo pandémico y pospandémico, destacando que es "necesario enseñar en escuelas y universidades para comprender al ser humano y enseñar a los niños y jóvenes a afrontar las incertidumbres". Y además destaca que: "Antes pensábamos que había cierto progreso y ahora el futuro es angustia"(p.2) .

En el curso de estos pensamientos, Morin (2020) destaca la importancia de que los niños y jóvenes aprendan a lidiar con sus miedos, ansiedades e incertidumbres, y al mismo tiempo alerta sobre la necesidad de un nuevo orden educativo, un nuevo don, donde la educación es el motor y el principal dispositivo para hacer frente a la angustia/incertidumbre.

De esta forma, este autor destaca también que la situación actual pone de manifiesto una nueva realidad que aspira a generar una conciencia transformadora de la sociedad, en la que las personas se reconozcan como parte de los problemas, pero sobre todo como parte de las soluciones. Es un momento de solidaridad y empatía, por eso, es necesario que los gobiernos desarrollen políticas educativas considerando los contextos, las múltiples identidades, saberes y singularidades que configuran a las personas involucrados en el acto educativo. 


\section{Metodología}

Este estudio es un extracto de la tesis doctoral, "Educación Permanente en Salud, el Camino Pedagógico para Reinventar el Trabajo Docente", que se encuentra en desarrollo, vinculado el Departamento de Medicina de la Universidad de Valencia/España, en cotutela con la Salud Colectiva de Universidad de Brasilia/Brasil.

Es una investigación descritivo-exploratoria, de enfoque cualitativo, que utilizó como instrumento de investigación entrevistas individuales semiestructuradas por videoconferencia. Esta investigación busca conocer las percepciones de los profesores/as de educación básica en España y Brasil, sobre las repercusiones de la COVID-19 en la educación, así como en la práctica diaria de los profesores/as con sus estudiantes en el año 2020.

Según Minayo \& Sanches (1993) la investigación cualitativa trabaja con el universo de significados, motivos, aspiraciones, creencias, valores y actitudes, lo que corresponde a un espacio más profundo de las relaciones, de los procesos y de los fenómenos que no pueden ser reducidos a una fórmula matemática.

En esta perspectiva, entrevistamos a profesores/as de España y Brasil, de la red de educación básica, que estuvieran trabajando en el aula (virtual y/o presencial) en el año 2020. Fueron entrevistados a un total de 12 profesores(as): 6 de España y 6 de Brasil, de los cuales 8 fueron mujeres y 4 hombres, con edades comprendidas entre los 28 y los 51 años. La experiencia en la enseñanza de esos profesores(as) variaba entre los 3 y los 29 años y abarcan las tres etapas de la enseñanza básica (infantil, primaria y secundaria). Los nombres (ficticios) utilizados para los profesores/as están relacionados con ciudades de su actividad laboral.

Para el análisis de los resultados se utilizaron los principios del análisis de contenido de Bardin (2008), la organización de la codificación seguida de la elección de las unidades de registro a través del tema, y se representó el recorte de ideas a través de diferentes oraciones, que tenían significados para el objetivo elegido sobre las respuestas a las preguntas abiertas, de las que surgieron las categorías: 1) El "desierto digital” ; 2) Educación - Potencialidades y Necesidades.

\section{Discusión}

\section{Categoría - El "desierto digital”}

La escuela, como uno de los espacios sociales con mayor probabilidad de contaminación, por ser uno de más intercambios y movilidad de sujetos de diferentes edades, fue el primer espacio en el que se suspendieron las actividades presenciales, debido a la COVID-19. Con esto, las tecnologías se han convertido en los principales dispositivos destinados a mantener la conexión educativa.

La tecnología fue la gran aliada para minimizar los impactos negativos que el confinamiento social trajo a la educación. El sistema educativo cambió por completo sus rutinas. Las instituciones educativas, los estudiantes, los profesores/as y los padres aún se están adaptando a la nueva realidad de la enseñanza causada por el Coronavirus.

Con la pandemia, los profesores(as) fueron arrojados frente a la computadora y llevaron las aulas a sus casas, cambiando las pizarras y pupitres de la escuela por pantallas y aplicaciones digitales. El trabajo escolar y los quehaceres domésticos se fusionaron, la vida privada y la profesional no podían separarse y el trabajo se triplicaba: "me siento extremadamente sobrecargada e invadida también, tengo una hija que aún amamanto y tuve que amamantarla 
cuando estaba enseñando y además mi casa se ha convertido en un espacio público" (Brasília).

Aprender, en un tiempo récord, a utilizar las herramientas digitales para enseñar fue el primer desafío, luego tuvieron que aprender a lidiar con las limitaciones impuestas por la distancia, tuvieron que enfrentarse a las pantallas de las computadoras a menudo sin rostros, las cámaras apagadas y la ausencia de ausencia. "Nunca sabemos si los estudiantes están participando, y siempre existe la sensación de que no están participando" (Independencia).

Según los entrevistados, al principio fue bastante difícil para los profesores/as lidiar con las computadoras, el aula virtual, la clase grabada, la clase en vivo, los videos y varias tecnologías que se utilizaban y probaban como: Google Scholar, Meet, Zoom, WhatsApp, radio, televisión, entre otras; "Fue complicado, te echaban toda la responsabilidad, teníamos que aprender la tecnología por nuestra cuenta, la formación de los profesores(as) era sólo el registro e inicio de sesión en el sistema" (Manaos).

Muchos tuvieron que superar sus miedos, su timidez, sus inseguridades para atravesar el espacio digital rompiendo no sólo las barreras tecnológicas, sino también las psicológicas, según los profesores/as; "me siento como si estuviera en una prisión mental" (Brasilia). Todo esto con un cambio drástico en la rutina doméstica y con una complicación aún mayor, para los que tienen niños en casa; "hoy tengo que lidiar con hijos, abuela enferma y estudiantes, todos de un mismo sitio, es todo muy malo" (Brasília).

Además, con el trabajo digital era imposible formar vínculos con los niños, pero con los estudiantes de secundaria es posible, según algunos de los entrevistados: "No hay forma de que pueda tomar la mano de un niño a través de una pantalla para enseñarle a escribir, el aprendizaje a distancia para los niños es imposible" (Barcelona). "Con los alumnos de primaria fue difícil desarrollar actividades, pero con los de secundaria fue positivo" (Valencia).

Según los profesores(as) de Brasil y España, ellos están viviendo un momento totalmente nuevo en su vida profesional causado por la COVID-19. De un momento a otro, tuvieron que adaptarse para seguir dando sus clases desde su espacio privado, sus casas, generalmente sin la infraestructura ideal y sin la ayuda del Estado.

Muchos profesores(as) que acceden sólo por el móvil porque no tiene un ordenador y otros tienen que compartir el ordenador con sus hijos, según Brasília: "Todo lo que se está usando es de uso privado del Profesor; tecnología, energía e internet, mis gastos aumentaron, tuve que cambiar mi internet por una más potente y mi factura de energía aumentó mucho".

Sin embargo, la falta de estructura de las escuelas y las mala condiciones de acceso a las actividades a distancia por parte de los estudiantes en diferentes entornos sociales, dio lugar a una agudización de las desigualdades y un aumento de la exclusión en la educación. Situación que fue remarcada por los profesores entrevistados: "utilizamos redes sociales, aplicaciones, etc. Pero como tenemos muchos estudiantes que viven en zonas rurales, alrededor del 6\% no tiene acceso a ninguna tecnología" (Independencia).

En este contexto, según la UNESCO (2021) la pandemia ha agravado la exclusión educativa, especialmente de los estudiantes en entornos de vulnerabilidad social, un total de 258 millones de niños y jóvenes quedaron absolutamente excluidos de la educación durante el confinamiento, lo que equivale al $17 \%$ de la población en edad escolar en todo el mundo. En Brasil, según UNICEF (2020), el 17\% de los niños y adolescentes de entre 9 y 17 años viven en sus casas sin acceso a Internet, lo que demuestra la desigualdad en el acceso a la educación digital. 
Así, surge otra cuestión a simple vista, la desigualdad social, como una de las mayores limitaciones para el uso de las nuevas tecnologías. Otra barrera relevante, según los entrevistados, es la falta de habilidades y el desconocimiento de la mayoría de los profesores/as y estudiantes en manejo y conocimiento de las tecnologías: "Para mí, al principio fue muy difícil tratar con la tecnología, pero los estudiantes lo tomaron muy rápido y ahora están con mucha autonomía, exploran la tecnología con bastante facilidad, nosotros los profesores estamos aprendiendo de los alumnos" (Brasilia).

\section{Categoría - Educación - Potencialidades y Necesidades}

El sistema educativo en el escenario mundial, incluso antes de la pandemia, ya tenía necesidades y desafíos aún no resueltos. La pandemia lo que hizo fue aumentar el desgaste y los problemas, tal como expuso Valencia: "en la educación, hay cosas que ya no funcionaban bien antes de la pandemia, como la falta de capacitación digital de los profesores/as y la necesidad de conectarse más con la realidad de los alumnos y la comunidad, con lo social, con la naturaleza, y todo esto es posible".

En el escenario actual, muchos profesores/as se sienten exhaustos y ansiosos, incluso después del regreso a aula. "Con la mascarilla la cuestión auditiva se complica, porque el fonema se forma desde la boca (por ejemplo, en la palabra moto), lo auditivo y lo visual son necesarios para comprender más fácilmente el sonido de las palabras/sílabas" (Barcelona). Y Valencia complementó: "Es como gritar en el desierto y que nadie te escuche, no puedes trabajar sin enfermarte, por eso yo también me enfermé de la garganta, el médico dijo que es como si cantara opera durante horas y horas".

Además la actual pandemia ha puesto aún más de manifiesto la importancia de la educación y su papel en el contexto del desarrollo social, económico y cultural para una sociedad sostenible, tal como sostiene Boff (2020) en una clase magistral virtual, disponible en youtube: "Tenemos que aprender de las señales que nos da la tierra, la COVID-19 es la consecuencia de la falta de educación de la humanidad, es un problema de educación, y a partir de ahora la educación no puede ser la misma que antes".

Boff (2020) también advierte que debemos reinventarnos como humanos, "creo que el lugar y la mayor forma de recrear las nuevas relaciones y una nueva proyección de lo que es ser humano, hombre y mujer, es la educación, porque la educación forma a las personas. Vosotros, profesores/as, tenéis la extraordinaria misión de rediseñar otro tipo de habitante del planeta tierra".

Si la crisis, es una crisis de la educación, como afirma Boff (2020), es importante señalar que la transmisión histórica del conocimiento producido, de generación en generación, se ha convertido en fundamental para el desarrollo del ser humano. Esta transmisión histórica se ha producido de diversas maneras a lo largo de la historia, pero en especial, en la escuela, como tarea del profesor/a.

En esta misma dirección, Saviani (2010) afirma que la labor educativa es el acto de producir, directa e intencionadamente en cada individuo, la humanidad que es producida histórica y colectivamente por todos los hombres.

En esta perspectiva, el profesor/a asumió la enorme importancia de transmitir, reinterpretar y recrear con las nuevas generaciones los conocimientos ya producidos por la raza humana, siendo básicos para el desarrollo de la sociedad en general y la condición de la humanidad en cada una de ellas. Como manifiesta otro de los entrevistados: "Porque el ser humano es naturalmente sociable desde la época de los monos, las relaciones de solidaridad son importantes y para eso, necesitamos el ojo en el ojo, el estar juntos, los intercambios, esto que la escuela nos ofrece" (Brasília). 
La escuela como espacio de visión integral, de sistematización de conocimientos y afectos, hace que la educación generada en este espacio, sea la propia materialización del sentido de la vida. Y este sentido de la vida de ahora en adelante debe construirse principalmente a partir de los aprendizajes de esta pandemia, es decir, "tratar a la Naturaleza y a la Tierra con más cuidado, amor y respeto" (Boff, 2020).

En ese sentido, todo el equipo de la escuela, su desempeño y el tratamiento de los conocimientos son de fundamental importancia para delinear nuevos rumbos de la práctica pedagógica en esta nueva realidad educativa que se evidencia en el confinamiento de los cuerpos y la necesidad de sentimientos de empatía.

Trabajo en una escuela donde vivo con más de 20 nacionalidades diferentes. Es un desafío, pero me siento preparada, siempre estoy estudiando. Pero no es porque la escuela o el gobierno fomente o apoye esta preparación, sino por mi propia necesidad de saber cómo tratar con este contexto diverso (Sevilla).

Sevilla, alerta a los gobiernos sobre la importancia de la formación permanente y continua de los profesores, sujetos responsables de la realización del acto pedagógico. Ya que el aprendizaje del profesor/a en su vida cotidiana como ser histórico y socialmente contextualizado, puede ayudar en la definición de un nuevo orden educativo/pedagógico.

Entendemos que, debido a que el mundo ha cambiado, la educación ya no es la misma, necesita ser recreada y los profesores(as) tendrán que estudiar más, saber más, relacionarse más, intercambiar más conocimientos, socializar más y extrapolar la información y el conocimiento. Es decir, desarrollar la "razón sensible", a fin de satisfacer las nuevas necesidades educativas y de vida impuestas por el Coronavirus.

Así, por ejemplo, Sevilla lo expresa claramente diciendo: “No entiendo la educación sin socialización, el aprendizaje es motivación social, somos emociones y si no trabajamos relacionándonos, no aprendemos".

En esta perspectiva, este mismo entrevistado advierte que debemos considerar al profesor como un aprendiz/estudiante/trabajador que inevitablemente necesita estar en un proceso constante de formación que incluye las dimensiones de inclusión, integración e internacionalización del conocimiento, y que permite la reinvención de su práctica en acto. Porque la práctica de la enseñanza, exige una amplia comunicación, información, socialización y correspondencia con las necesidades sociales, procesos de participación y conexión contigo y con el otro (Vieira, 2013).

Según un otro entrevistado: "En los colegios hay mucha diversidad, es importante discutir y promover acciones de internacionalización del conocimiento en las escuelas, porque los alumnos son el futuro y muchos que terminan el bachillerato no van a la universidad por sus condiciones económicas" (Barcelona). Así, entendemos que, la internacionalización del conocimiento en el espacio escolar es intrínseca a las necesidades pedagógicas que impregnan este espacio.

La internacionalización del conocimiento en el diccionario significa captar la información de manera amplia, en todos los sentidos. Eso nos lleva a reflexionar más sobre la importancia de la información en la visión de Morin (2008, p. 6), que advierte que "hoy en día estamos condenados al conocimiento ciego, porque a pesar de la multiplicación de la información y el conocimiento, sobre todo, sólo entendemos fragmentos separados". 
Todo ha cambiado de perspectiva, hay que pensar mucho, hay que tener mucho cuidado de no generar más esfuerzo a partir de ello. Estamos aprendiendo mucho más, nuestra práctica cambiará con el aprendizaje digital, que ahora es una tormenta, pero es un aprendizaje que ayudará más tarde cuando volvamos al aula. Hay momentos en el que uno no lo logra, uno no sabe cómo hacer esto o aquello. Pero intercambiamos conocimientos, uno ayuda al otro (Brasilia).

Brasilia, dialoga con Morin (2008) cuando trae a escena la importancia del intercambio de conocimientos y aprendizajes relacionados con la práctica. Y en esa misma dirección, Bondía (2002 p. 22) dice que: "hay un énfasis contemporáneo en la información, en estar informado y esta intensa búsqueda de información y conocimiento, no significa necesariamente, un conocimiento en el sentido de "sabiduría", sino en el sentido de estar informado".

Las experiencias, en su contexto general, siempre abren nuevas visiones. "La experiencia con la clase en línea/remota fue muy diferente de algo a lo que estaba acostumbrada, pero descubrí muchas posibilidades, como el contacto más directo con las familias" (Sevilla).

En esa perspectiva, entendemos que el conocimiento es una obra abierta que se cierra con el hecho científico y la experiencia y aun así, con posibilidades de nuevas reaperturas y que la información por sí misma no es sinónimo de conocimiento o experiencia.

Para obtener todos los sentidos y para que los fragmentos de conocimiento y de saber se unan, para generar conocimiento y su internacionalización, es necesario conectarse con el hecho científico y pasar por el proceso de la experiencia, que según Bondía (2002 p. 23), "la experiencia es lo que nos pasa, lo que nos sucede, lo que nos toca. No lo que sucede, no lo que pasa, o lo que toca".

Desde esta perspectiva, Ceccim (2005, p. 10) contribuye con la concepción de Educación Permanente en salud (EPS), lo que para él sucede todo el tiempo en el mundo del trabajo, "ya sea a través del intercambio de conocimientos, estos no sólo técnicos y científicos, sino también las dimensiones éticas de la vida, o a través de las relaciones". En ese sentido, estos procesos deben estar guiados por la inseparabilidad entre la formación, la gestión, la atención, la participación, definida como cuadrilátero da formación en salud (Ceccim \& Feuerwerker, 2004).

Uno de los entrevistados/as dialoga con los autores arriba citados al traer al contexto de la educación la necesidad de la interculturalidad e internacionalización del conocimiento en el espacio escolar, así como la necesidad de formación de los profesores/a en la perspectiva del aprendizaje en el escenario del conocimiento del otro y los intercambios interculturales: "La base del alumno es su conocimiento, su lenguaje y su cultura y necesitamos conocer este contexto primero para luego poder enseñarles algo" (Manaos).

\section{Conclusiones}

En esta investigación, tanto los profesores brasileños como los españoles vivieron momentos de gran sufrimiento, envueltos por la metáfora del "desierto" digital. Los profesores tuvieron que adaptarse a las nuevas tecnologías, a la distancia de los cuerpos y a trabajar a través de pantallas digitales muchas veces sin poder ver las caras de los estudiantes y sin saber realmente si estos estaban siguiendo la clase. Además, los profesores tuvieron que superar miedos y carencias en relación a las tecnologías a emplear y sus temores a ser contagiados durante las clases presenciales, todo ello en una situación de precariedad laboral. 
Pero la mayoría de los entrevistados|as, expresaron sentimientos de solidaridad y son conscientes de la importancia de su rol y el papel social que juega la educación en la vida de miles de estudiantes y familias y harán todo lo que esté a su alcance para ayudar a sus estudiantes de la mejor manera posible.

La escuela y la educación escolar ya no son las mismas, incluso después del retorno a las clases el contexto educativo se ha vuelto más complejo, otros desafíos se han añadido a los ya existentes, como las condiciones de trabajo que se han vuelto mucho peores, el manejo de sus propios temores/emociones de pérdidas y el desafío de reavivar la llama de los estudiantes que están desanimados, enfermos y sin estímulo.

Por un lado, la nueva realidad educativa se evidencia en el distanciamiento en los procesos de enseñanza y aprendizaje, entre las generaciones de tecnologías digitales (estudiantes) y las de tecnologías analógicas (profesores/as) y la distancia de los cuerpos provocada por la necesidad de un confinamiento y el uso de equipos de protección como la mascarilla, que dificulta la práctica de la enseñanza, la constitución de vínculos y el aprendizaje de los niños y jóvenes.

Por otra parte, se hace imperativa la reafirmación de la importancia del papel del profesor/a como protagonista del acto de educar y la necesidad de desarrollar la "razón sensible" para una práctica docente más humanizada. Los procesos formativos de los docentes y la propia educación deben ser un compromiso ético, estético y político de toda sociedad, pero especialmente por los gobiernos, y deben estar guiados por la inseparabilidad entre la formación, la gestión, la atención, la participación (Ceccim\&Feuerwerker,2004), la interculturalidad, la intersectorialidad, la inclusión, internacionalización del conocimiento y el proyecto político pedagógico de la escuela.

\section{Referencias}

Bardin, L. (2008). Análise de Conteúdo de Laurence Bardin (Vol. 70). Edições 70.

Boff, L. (2020, 20 setembro) Aula Magna: Educação \& Cidadania com Leonardo Boff. [Vídeo] YouTube. https://www.youtube.com/watch?v=PiNOsoWgq9w

Bondía, J. L. (2002). Notas sobre a experiência e o saber de experiência. Revista Brasileira de Educação, 19, 20-28. https://doi.org/10.1590/s1413-24782002000100003

Ceccim, R. B. (2005). Educação Permanente em Saúde: descentralização e disseminação de capacidade pedagógica na saúde. Ciência \& Saúde Coletiva,10(4), 975-986. https://doi.org/10.1590/\$1413-81232005000400020

Ceccim, R. B., y Feuerwerke, L. C. M. (2004). O quadrilátero da formação para a área da saúde: ensino, gestão, atenção e controle social. Physis: Revista de Saúde Coletiva, 14(1), 4165. https://doi.org/10.1590/s0103-73312004000100004

España. Real Decreto $n^{\circ} 463$ (2020, 14 de marzo), por el que se declara el estado de alarma para la gestión de la situación de crisis sanitaria ocasionada por la COVID-19. Ministerio de la Presidencia, Relaciones con las Cortes y Memoria Democrática Española BOE $n^{\circ} 67$.

España. Real Decreto $n^{\circ} 555$ (2020, 5 de junio), por el que se prorroga el estado de alarma declarado por el Real Decreto 463/2020, de 14 de marzo, por el que se declara el estado de alarma para la gestión de la situación de crisis sanitaria ocasionada por la COVID-19. Ministerio de la Presidencia, Relaciones con las Cortes y Memoria Democrática Española BOE nº 2022. 
ILO. (2020, 29 April) ILO Monitor: COVID-19 and the world of work. Third edition. Updated estimates and analysis.

FENEP. (2020). Portal. https://www.fenep.org.br/

Minayo, M. C. S. y Sanches, O. (1993). Quantitativo-qualitativo: oposição ou complementaridade? Cadernos de Saúde Pública, 9(3), 237-248. https://doi.org/10.1590/s0102-311×1993000300002

Morin, E. (2020). Lições da pandemia: o despertar para as grandes verdades humanas. Fronteiras do Pensamento. https://www.fronteiras.com/artigos/licoes-da-pandemiao-despertar-para-as-grandes-verdades-humanas

Morin, E. (2008). Introdução ao Pensamento Complexo. 5. ed. Lisboa: Instituto Piaget.

OMS. (2020, 11 marzo). Organización Mundial de la Salud. https://www.who.int/es/directorgeneral/speeches/detail/who-director-general-s-opening-remarks-at-the-mediabriefing-on-covid-19--11-march-2020

Said, F. (2020, 25 marzo). Em meio à pandemia, Bolsonaro quer crianças de volta à escola. Congresso em Foco. https://congressoemfoco.uol.com.br/governo/bolsonarocontraria-autoridades-de-saude-e-pede-fim-do-confinamento-em-massa/

Saviani, D. (2010). Interlocuções pedagógicas: conversa com Paulo Freire e Adriano Nogueira e 30 entrevistas sobre educação. Campinas: Autores Associados.

UNESCO. (2020, 28 abril). El aprendizaje nunca se detiene - Cuéntele a la UNESCO cómo hace frente al cierre de las escuelas durante la crisis del COVID-19. https://es.unesco.org/news/aprendizaje-nunca-se-detiene-cuentele-unesco-comohace-frente-al-cierre-escuelas-durante-crisis

UNESCO (2021, 05 enero). Coalición Mundial para la Educación COVID-19. https://es.unesco.org/covid19/globaleducationcoalition.

Vieira, M. E. M. Programa Saúde na Escola: a Intersetorialidade em Movimento. Dissertação de mestrado, UnB, 2013. https://repositorio.unb.br/handle/10482/14859. 